\title{
Exploring management of antenatally diagnosed fetal syphilis infection
}

\author{
Margot Rosenthal ${ }^{1 *}$, Vanessa Poliquin ${ }^{1}$
}

\begin{abstract}
Background: The incidence of syphilis among Canadian women of childbearing age has risen dramatically in the past decade, with a resurgence of infants born with congenital syphilis. While guidelines exist to guide maternal infection during pregnancy, there is little evidence available to guide management in situations where the developing fetus is found to be severely affected.

Case review: Our patient presented in the second trimester of her pregnancy as syphilis contact. Positive serologic tests (venereal disease research laboratory titre of 1:64) and a chancre suggested primary infection. Ultrasound demonstrated a fetus at 19+3 weeks gestation with hydrops fetalis and a markedly abnormal brain. Amniocentesis confirmed congenital syphilis infection on polymerase chain reaction testing. After nine days of intravenous penicillin $\mathrm{G}$, the fetal status had worsened, and the family ultimately chose a medical termination of the pregnancy.
\end{abstract}

Discussion: Evolving ultrasound technology has allowed us to identify severely affected fetuses, who may historically have been delivered stillborn. Following routine syphiliotherapy with benzathine penicillin, these abnormal ultrasound features may take weeks or months to reverse, which poses a challenge in prognostication and counselling. Case reports data suggests intensive treatment with intravenous penicillin may be effective in severe cases where fetal hydrops is present.

Conclusion: This case highlights the potential morbidity of fetal syphilis infection and underscores the paucity of current literature. Information sharing will be essential to build a modern knowledge base on treating this ancient disease.

Suggested citation: Rosenthal MA, Poliquin V. Exploring management of antenatally diagnosed fetal syphilis infection. Can Commun Dis Rep 2022;48(2/3):111-4. https://doi.org/10.14745/ccdr.v48i23a09

Keywords: syphilis, fetal syphilis, pregnancy, ultrasound findings

\section{Introduction}

Syphilis infections among Canadian women of childbearing age has risen dramatically in the past decade, with resurgence of affected pregnancies and infants born with congenital syphilis (1). Vertical transmission from transplacental passage of spirochetes occurs in $50 \%-80 \%$ of untreated patients, with transmission rates highest with primary and secondary infections (2). Affected gravidas are at increased risk of stillbirth and preterm delivery (3). Antenatal treatment with intramuscular benzathine penicillin $G$ is highly effective at preventing congenital syphilis (4). In a minority of cases, sequelae of fetal infection can be detected by ultrasound (5). There is a paucity of evidence available to guide clinicians caring for these affected pregnancies.

\section{Case presentation}

We present the case of a 29-year-old G4P3 (fourth pregnancy; three deliveries) female called to care in her rural community in Manitoba, Canada as a syphilis contact. She presented pregnant with an unsure last menstrual period, with no significant medical or surgical history. Given her risk as a sexual contact, she was treated for syphilis empirically with one dose of intramuscular benzathine penicillin $\mathrm{G}$. She complained of worsening back pain and weakness over several weeks with increasing difficulty moving her neck, prompting transfer to our tertiary care setting for further evaluation and management.

A painless, shallow ulcerated vulvar lesion was identified at the posterior fourchette, which was swabbed and ultimately positive for Treponema pallidum (T. pallidum) deoxyribonucleic acid on 
polymerase chain reaction (PCR) testing. Her clinical symptoms raised concern for neurosyphilis as she had a positive Jolt test suggesting meningeal irritation although Brudzinsky and Kernig's signs were negative. Strength in the lower extremities was objectively decreased. She did not have a fever or skin rash.

She was admitted to the antepartum unit at Winnipeg's Health Sciences Centre and co-managed by the Infectious Disease, Reproductive Infectious Diseases and Maternal Fetal Medicine services. A lumbar puncture was performed, and antibiotic therapy initiated with intravenous penicillin G (24 million units per day divided every four hours in accordance with neurosyphilis dose recommendations). The cerebrospinal fluid showed low protein $(0.12 \mathrm{~g} / \mathrm{L})$ and normal glucose levels, with a normal total nucleated cell count of $1 \times 10^{6} / \mathrm{L}$. Uninfused computed tomography and magnetic resonance imaging scans of the brain and spine (done without gadolinium as it is contraindicated in pregnancy) did not demonstrate acute abnormalities. She had microcytic anemia with a hemoglobin level of $77 \mathrm{~g} / \mathrm{L}$ and inflammatory markers with an erythrocyte sedimentation rate higher than $140 \mathrm{~mm} /$ hour and a C-reactive protein level of $58 \mathrm{mg} / \mathrm{L}$.

Maternal syphilitic infection was confirmed with serum Venereal Disease Research Laboratory (VDRL) titre of 1:64 and T. pallidum IgG detected by chemiluminescent microparticle immunoassay. In addition to the positive PCR result on the vulvar lesion confirming a chancre, a review of serology from a year prior confirmed this to be a primary infection. No other sexually transmitted infections were identified, and additional serologic findings are outlined in Table 1. The cerebrospinal fluid was negative for VDRL and all cultures (bacterial, mycobacterial and fungal) were negative.

Table 1: Maternal investigations

\begin{tabular}{|l|l|l|}
\multicolumn{1}{|c|}{ Test } & \multicolumn{1}{c|}{ Source } & \multicolumn{1}{c|}{ Result } \\
\hline $\begin{array}{l}\text { Treponema pallidum Ab } \\
\text { IgG+IgM }\end{array}$ & Serum & Positive \\
\hline VDRL & Serum & Reactive 1:64 \\
\hline $\begin{array}{l}\text { Toxoplasma gondii } \\
\text { IgM/IgG }\end{array}$ & Serum & Negative/negative \\
\hline Cytomegalovirus IgM/lgG & Serum & Negative/antibody detected \\
\hline Parvovirus B19 IgM/lgG & Serum & Negative/indeterminate \\
\hline West Nile virus IgM & Serum & Negative \\
\hline $\begin{array}{l}\text { Anaplasma } \\
\text { phagocytophilum IgG }\end{array}$ & Serum & Negative \\
\hline $\begin{array}{l}\text { Treponema pallidum DNA } \\
\text { PCR/NAAT }\end{array}$ & $\begin{array}{l}\text { Vulvar } \\
\text { lesion }\end{array}$ & Target detected \\
\hline CSF-VDRL & CSF & Non-reactive \\
\hline $\begin{array}{l}\text { Treponema pallidum DNA } \\
\text { PCR/NAAT }\end{array}$ & CSF & Target not detected \\
\hline Bacterial culture & CSF & Negative \\
\hline Mycobacterial culture & CSF & Negative \\
\hline Fungal culture & CSF & Negative \\
\hline $\begin{array}{l}\text { Abbreviations: CSF, cerebrospinal fluid; DNA PCR/NAAT, deoxyribonucleic acid polymerase } \\
\text { chain reaction/nucleic acid amplification test; IgM/lgG, immunoglobulin M/immunoglobulin G; } \\
\text { VDRL, Venereal Disease Research Laboratory }\end{array}$ & \\
\hline
\end{tabular}

Page 112 CCDR • February/March $2022 \bullet$ Vol. 48 No. 2/3
Ultrasound performed by Maternal Fetal Medicine demonstrated a single live intrauterine pregnancy at gestational age of $19+3$ weeks. The fetus was affected by severe hydrops fetalis, as demonstrated by large pleural effusions, large ascites compressing the kidneys and body wall edema (Figure 1). The brain was very abnormal with bright cerebrospinal fluid and streaky echoes throughout the cortex (Figure 2). There was polyhydramnios with a maximum vertical pocket of $8 \mathrm{~cm}$, with a thickened and overtly hydropic placenta (Figure 3). The umbilical artery Doppler ultrasound was abnormal with an increased systolic to diastolic ratio; however, the middle cerebral artery (MCA) Doppler did not demonstrate evidence of fetal anemia (peak systolic velocity 1.37 MoM). To complete the evaluation for hydrops, amniocentesis was performed and sent for microbiologic and genetic evaluation. Results are summarized in Table 2 and were notably positive for T. pallidum on PCR testing.

Figure 1: Very large ascites, echogenic clumped bowel, compressed kidneys suggestive of high intra-abdominal pressure at $19+3$ weeks gestational age

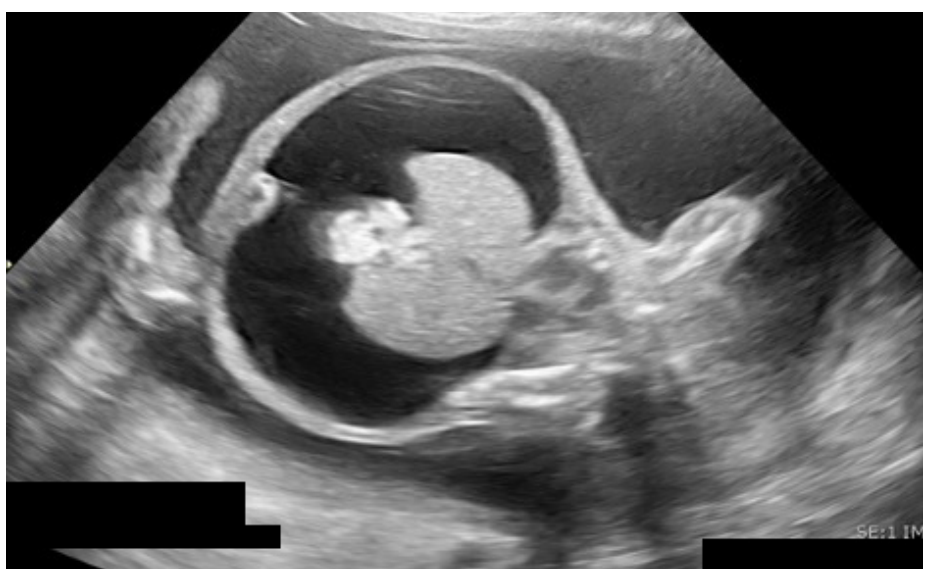

Figure 2: Marked scalp edema, very abnormal brain with bright cerebrospinal fluid, streaky echoes throughout brain matter at $19+3$ weeks gestational age

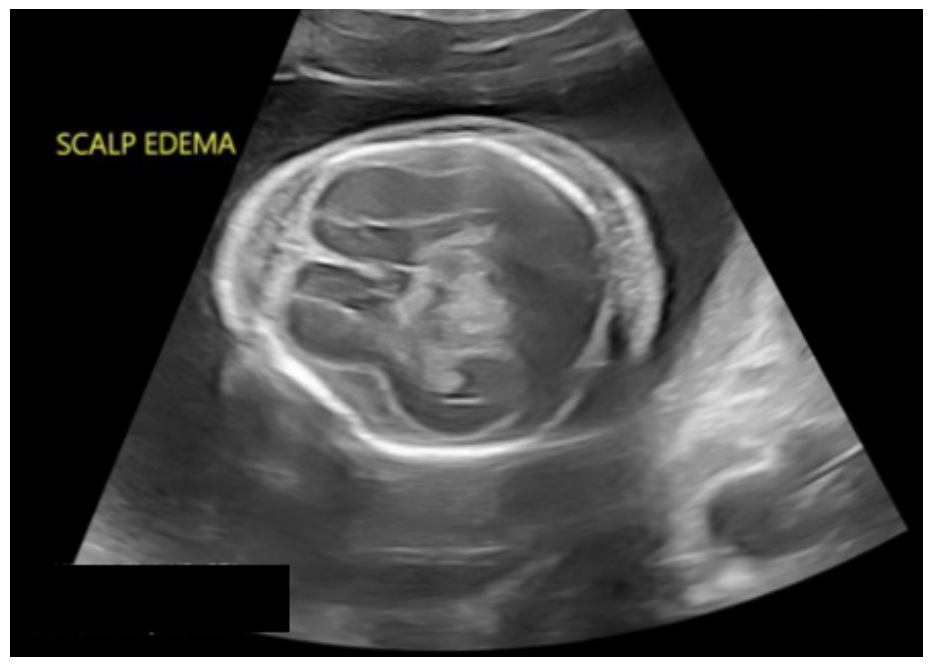


Figure 3: Enlarged, hydropic, placenta with multiple cystic lesions at $19+3$ weeks gestational age

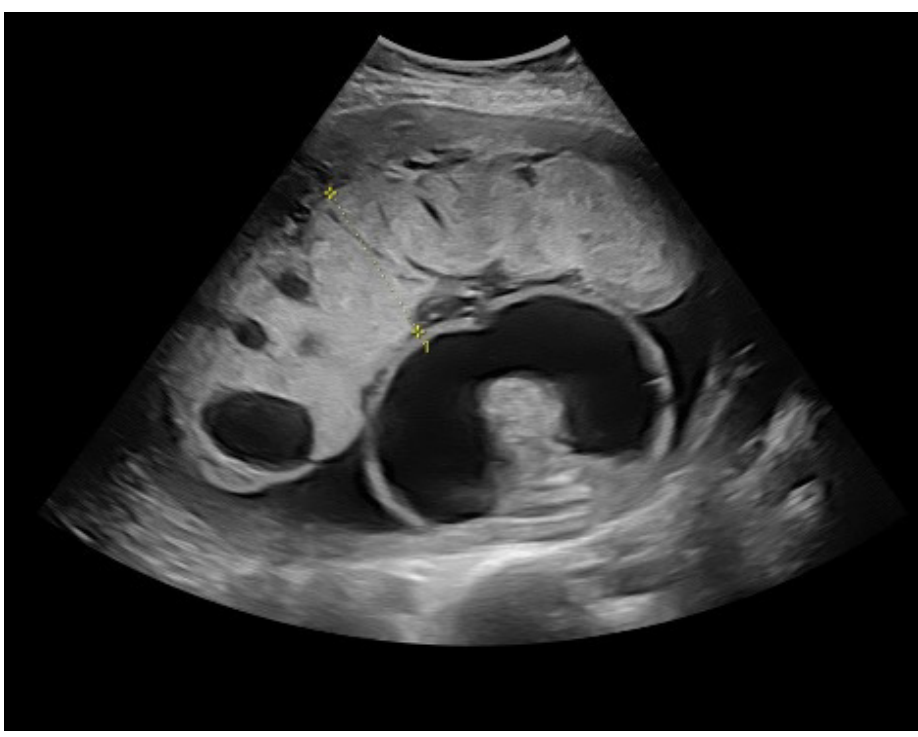

Table 2: Amniocentesis results

\begin{tabular}{|l|l|l|}
\multicolumn{1}{|c|}{ Test } & \multicolumn{1}{c|}{ Source } & \multicolumn{1}{c|}{ Result } \\
\hline $\begin{array}{l}\text { Treponema pallidum } \\
\text { DNA PCR/NAAT }\end{array}$ & Amniotic fluid & Target detected \\
\hline $\begin{array}{l}\text { Cytomegalovirus DNA } \\
\text { NAAT }\end{array}$ & Amniotic fluid & Target not detected \\
\hline $\begin{array}{l}\text { Parvovirus B19 DNA } \\
\text { NAAT }\end{array}$ & Amniotic fluid & Target not detected \\
\hline Viral culture & Amniotic fluid & Negative \\
\hline
\end{tabular}

Abbreviations: DNA NAAT, deoxyribonucleic acid nucleic acid amplification test;

DNA PCR/NAAT, deoxyribonucleic acid polymerase chain reaction/nucleic acid amplification test

Treatment was continued with intravenous penicillin in the hopes of achieving sufficient levels to treat the fetal infection. After nine days, a repeat detailed ultrasound demonstrated worsening fetal ascites. The fetus was now $20+5$ weeks gestation with an abdominal circumference of $282 \mathrm{~mm}(32+2$ weeks size $)$

(Figure 4). The scalp edema and Doppler ultrasound results remained stable. The family was counselled in depth about options, including termination of pregnancy or expectant management with serial ultrasound and fetal magnetic resonance imaging to follow structural neurologic changes. Overall, the prognosis was thought to be poor given the extent of hydrops at early gestation and severity of the changes in the cerebral cortex. Ultimately, the family decided to proceed with termination of pregnancy. Medical induction of labour was carried out with mifepristone followed by vaginal misoprostol. A stillborn hydropic female infant was delivered, indicative of intrapartum demise, at $21+0$ weeks gestation and weighing $747 \mathrm{~g}$ (greater than $99^{\text {th }}$ centile for gestational age). The family declined an autopsy. The placenta weighed $387 \mathrm{~g}$ (over twice the average weight for that gestational age) with pathologic signs of decidual hemorrhage, necrosis and acute inflammation.
Figure 4: Worsening of ascites with abdomen measuring 32 weeks size at $20+5$ weeks gestational age

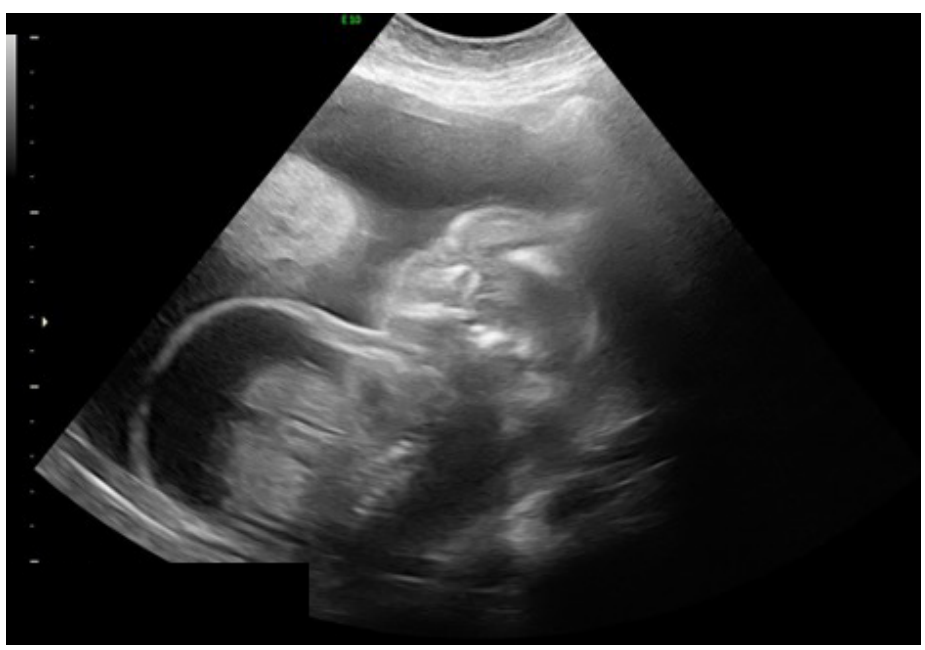

Maternal symptoms continued to improve after delivery and intravenous penicillin was discontinued. Once able to ambulate without assistance, she was discharged home. Follow-up serology showed an appropriate treatment response with her VDRL titre down to 1:4.

\section{Discussion}

Congenital syphilis is a preventable disease. In a 2019 statement, the Society of Obstetricians and Gynecologists of Canada advocated for adequate screening in pregnancy, with enhanced three-point screening in outbreak areas (1). Treatment of syphilis in pregnancy has been shown to be highly effective at decreasing rates of congenital syphilis, syphilitic stillbirth and obstetrical complications such as preterm birth (3). The treatment of choice is intramuscular benzathine penicillin $G$ at a dose of 2.4 million units, with a second dose administered one week later (3). A third dose in recommended for patients with late latent syphilis (3).

Given the increased risk for intrauterine growth restriction, fetal anomalies, and hydrops fetalis, additional sonographic surveillance is recommended (6). In a cohort study of seropositive women who had an ultrasound prior to treatment for syphilis, Rac et al. found that $30 \%$ had evidence of fetal syphilis (5). Fetal hepatomegaly was the most noted abnormality (seen in $79 \%$ of affected fetuses) with placentomegaly, polyhydramnios, ascites and evidence of fetal anemia with elevated peak systolic velocity in the MCA Doppler ultrasound was also seen (5). The authors went on to track timeline to resolution of abnormal Doppler ultrasound features and found MCA Doppler ultrasound abnormalities, ascites and polyhydramnios were the first to resolve ( $80 \%$ resolution within 40 days), followed by placentomegaly and hepatomegaly, which could persist until delivery. It should be noted that ultrasound findings of congenital syphilis are not commonly identified before 18 weeks gestation 
as the immature fetal immune system is not yet able to mount a sufficient response $(3,6)$.

Congenital syphilis was historically diagnosed after birth, with most infected neonates showing symptoms by the second month of life (6). Modern technologies, including ultrasound, have demonstrated an evolving role in diagnosis of fetal syphilis over the last three decades. Much of the clinical wisdom in treating syphilis predates these antenatal diagnostics, leaving the modern clinician with a diagnosis but limited evidence to guide treatment.

In our patient's case, treatment was initiated using intravenous (IV) penicillin G at neurosyphilis dosing for maternal indication. Treatment was continued in hopes of treating the fetal infection. A case report by Galan et al. described the use of IV penicillin G to treat syphilis-related fetal hydrops at 24 weeks gestation after an ultrasound showed worsening features 10 days after receiving intramuscular benzathine penicillin (7). Their patient showed complete resolution of sonographic findings after 10 days of IV therapy (7). A second case by Chen et al. reported on a patient diagnosed with secondary syphilis at 28 weeks gestation with sonographic findings of fetal hydrops and anemia and with an elevated MCA peak systolic velocity (8). Their patient was admitted and treated with IV penicillin $\mathrm{G}$ for 14 days to target maximal and sustained fetal levels (8). The patient underwent an ultrasound-guided cordocentesis and fetal transfusion, and showed resolution of the fetal hydrops within two weeks (8). Although both cases had relatively rapid resolution of symptoms when compared with the data from Rac's cohort, there is not enough information to draw conclusions about optimal therapy for fetal syphilis.

Our case outlines a situation that, without treatment, would almost certainly would have progressed to syphilitic stillbirth. Current obstetrical guidelines for investigation of stillbirth are vague in the recommendations for infectious screening, with many local hospitals developing their own protocols (9). This leads to possibility of missed diagnoses of syphilis during investigation of non-immune fetal hydrops and stillbirth, with implications at the level of both patients and greater public health.

\section{Conclusion and call to action}

This case highlights the potential severity of fetal syphilis infection and underscores the paucity of current literature to guide management of a severely affected fetus in the antepartum period. Information sharing will be essential to build a modern knowledge base on the treatment of this resurging infection. We urge obstetrical care providers to increase infectious screening and keep syphilis on the differential when investigating severe complications.

\section{Authors' statement}

The authors have no conflicts of interest to disclose.

The content and view expressed in this article are those of the authors and do not necessarily reflect those of the Government of Canada.

\section{Competing interests}

None.

\section{Acknowledgements}

The authors wish to thank Dr. C Schneider for her contribution to this case.

\section{Funding}

This publication was not funded.

\section{References}

1. The Society of Obstetricians and Gynaecologists of Canada. SOGC Infectious Diseases Committee Statement on Syphilis. Ottawa (ON): SOGC; 2019. https://sogc.org/en/content/featured-news/sogcinfectious-diseases-committee-statement-on-syphilis.aspx

2. Gomez GB, Kamb ML, Newman LM, Mark J, Broutet N, Hawkes SJ. Untreated maternal syphilis and adverse outcomes of pregnancy: a systematic review and meta-analysis. Bull World Health Organ 2013;91(3):217-26. DOI PubMed

3. Adhikari EH. Syphilis in Pregnancy. Obstet Gynecol 2020;135(5):1121-35. DOI PubMed

4. Alexander JM, Sheffield JS, Sanchez PJ, Mayfield J, Wendel GD Jr. Efficacy of treatment for syphilis in pregnancy. Obstet Gynecol 1999;93(1):5-8. DOI PubMed

5. Rac MW, Bryant SN, Mclntire DD, Cantey JB, Twickler DM, Wendel GD Jr, Sheffield JS. Progression of ultrasound findings of fetal syphilis after maternal treatment. Am J Obstet Gynecol 2014;211(4):426.e1-6. DOI PubMed

6. Rac MW, Stafford IA, Eppes CS. Congenital syphilis: A contemporary update on an ancient disease. Prenat Diagn 2020;40(13):1703-14. DOI PubMed

7. Galan HL, Yandell PM, Knight AB. Intravenous penicillin for antenatal syphilotherapy. Infect Dis Obstet Gynecol 1993;1(1):7-11. DOI PubMed

8. Chen I, Chandra S, Singh A, Kumar M, Jain V, Turnell R. Successful outcome with intrauterine transfusion in non-immune hydrops fetalis secondary to congenital syphilis. J Obstet Gynaecol Can 2010;32(9):861-5. DOl PubMed

9. Leduc L. No. 394-stillbirth Investigation. J Obstet Gynaecol Can 2020;42(1):92-9. DOI PubMed 